\title{
Representaciones sociales acerca de la lucha, en Chile, por el derecho a la salud*
}

\author{
Social representations of the fight for the right to health in Chile
}

Representações sociais atinentes na luta, no Chile, pelo direito à saúde

\begin{abstract}
Pedro Enrique Villasana-López'; Eva Carolina Álvarez-González².
1 Médico, Universidad de Carabobo (Venezuela). Phd Ciencias Universidad de Carabobo (Venezuela). Docente-investigador Departamento de Salud, Universidad de Los Lagos. Puerto Montt. Chile. ORCID: https://orcid.org/0000-0001-8713-8202.pedro.villasana@ulagos.cl

2 Licenciada en Sociología, Universidad Nacional de Cuyo, Mendoza (Argentina). Especialista en Gestión en Salud Pública, Instituto de Altos Estudios "Dr. Arnoldo Gabaldón", Maracay (República Bolivariana de Venezuela). Investigadora del Instituto de Estudios Críticos, Santiago de Chile (Chile). evacaroalvarez@gmail.com
\end{abstract}

Recibido: 12/11/2018. Aprobado:15/05/2019. Publicado: 01/10/2019

Villasana-López PE, Álvarez-González EC. Representaciones sociales acerca de la lucha, en Chile, por el derecho a la salud. Rev. Fac. Nac. Salud Pública. 2019;37(2):44-53. Dor:10.17533/udea.rfnsp.v37n3a06

\section{Resumen}

Objetivo: El presente trabajo explora las representaciones que, acerca de la lucha por el derecho a la salud, tienen algunos actores sociales que participan de acciones colectivas por la salud en Santiago de Chile. Metodología: La aproximación al fenómeno se realiza desde el paradigma pospositivista, con enfoque cualitativo; la información fue obtenida a partir de nueve entrevistas focalizadas, y su análisis desde la teoría fundamentada. Resultados: La lucha por el derecho a la salud se refiere a la defensa de la vida en forma integral, entendiéndola como el esfuerzo por la transformación de la sociedad a partir de la construcción colectiva de un nuevo modelo económico-político. Conclusión: Esta aproximación permite hacer visible que los actores que hacen parte de las acciones colectivas no conforman un movimiento unificado ni estructurado, y que las luchas por el derecho a la salud constituyen un fenómeno emergente, que puede enmarcarse en un proceso de repolitización de la sociedad chilena, en cuyo seno las representaciones acerca de las luchas por el derecho a la salud de los actores sociales investigados tendrían un carácter emancipatorio, con base en su perfil contrahegemónico.

-Palabras clave: derecho a la salud, representaciones sociales, salud colectiva, Chile.

* El presente artículo se basa en trabajo especial de grado para optar al título de especialista en Gestión en Salud Pública del Instituto de Altos Estudios “Dr. Arnoldo Gabaldón”, Maracay, República Bolivariana de Venezuela. Presentado y aprobado el 9 de marzo de 2018. Disponible en biblioteca. 


\begin{abstract}
Objective: This article explores the representations that some social actors participating in collective actions for health in Santiago de Chile have with respect to the fight for the right to health. Methodology: The phenomenon is explored from a post-positivist paradigm with a qualitative approach. The information was gathered from nine focused interviews and their analysis was based on grounded theory. Results: The fight for the right to health refers to the defense of life in a holistic way, understanding it as an effort towards the transformation of society through the collective construction of
\end{abstract}

a new economic-political model. Conclusion: This approach shows that social actors that are part of collective actions do not form a structured or cohesive movement. Also, fights for the right to health constitute an emerging phenomenon that may be seen as part of a repolitization of the Chilean society, in which representations of the fights for the right to health in the investigated actors would be of an emancipatory in nature, based on their counter-hegemonic profile.

--------Keywords: Right to health, social representations, collective health, Chile

\section{Resumo}

Objetivo: O presente trabalho explora as representações que, atinentes na luta pelo direito à saúde, possuem alguns atores sociais que participam de ações coletivas para a saúde em Santiago, Chile. Metodologia: A abordagem do fenômeno é realizada a partir do paradigma pós-positivista, com abordagem qualitativa; as informações foram obtidas de nove entrevistas focadas e sua análise a partir da teoria fundamentada. Resultados: A luta pelo direito à saúde refere-se à defesa da vida de forma integral, entendendo-a como o esforço de transformação da sociedade a partir da construção coletiva de um novo modelo econômico-político. Conclusão: Essa abordagem permite tornar visível que os atores que fazem parte das ações coletivas não constituem um movimento unificado ou estruturado e que as lutas pelo direito à saúde constituem um fenômeno emergente, que pode ser emolduradorado num processo de repolitização no colo da sociedade chilena, em que as representações atinentes nas lutas pelo direito à saúde dos atores sociais apuradosados teriam caráter emancipatório, com base em seu perfil contra-hegemônico.

--Palavras-chave: direito à saúde, representações sociais, saúde coletiva, Chile

\section{Introducción}

El sistema de salud público en Chile se articuló mediante la implementación, en 1952, de la Ley 10383, presentada por Salvador Allende, con la cual se creó el Servicio Nacional de Salud (SNS), durante el gobierno de Gabriel González Videla (1946-1952). A pesar de ser aprobada con modificaciones, esta ley cumplió con su objetivo principal: integrar, en un organismo dependiente del Ministerio de Salubridad, los servicios de salud en todo el territorio nacional [1].

Illanes [1] señala que la posibilidad de la aprobación de esta ley estuvo directamente relacionada con el peso de las acciones colectivas y la organización de pobladores urbanos y campesinos en torno al acceso a derechos sociales, que comenzaron a articularse desde la década de los cuarenta del siglo xx.

El sns prestaba servicio de salud a obreros (prevalece el esfuerzo físico en el desarrollo de tareas); por su parte, los empleados (prevalencia de trabajo intelectual) debían realizar aportes individuales al Servicio Médico Nacional de Empleados [2].

En 1969, mediante la Ley 16781, propuesta por el Colegio Médico (actor corporativo), se crea el Servicio
Médico Nacional de Empleados (Sermena), que institucionaliza la modalidad de libre elección, e implicó la fragmentación del Servicio Nacional de Salud.

El acalorado debate sobre la modalidad de libre elección radicalizó a los distintos estamentos de la salud, y así, empleados y obreros se cohesionaron, iniciando los años setenta en dos bloques: los partidarios de una salud pública-centralizada y aquellos que defienden el derecho a elegir libremente [3].

A partir del triunfo electoral de Salvador Allende en 1970, se llevaron a cabo acciones que transformaron el campo de la salud en Chile. Mediante modificaciones a la Constitución de 1925, se instituyó el rol del Estado como garante del ejercicio de la atención médica, preventiva, curativa y de rehabilitación [1]. Asimismo, se tomaron medidas tendientes a democratizar la gestión de la salud, con la creación de tres organismos de participación territorial: consejos locales de salud, consejos locales de áreas de salud y consejos paritarios, con representatividad de trabajadores de la salud y la población. Illanes señala que la salud pasó "a ser un tema y una acción que comprometía el interés de toda la sociedad; es decir, que formaba parte de la construcción global de un nuevo proyecto" [1, p. 478]. 
Para aquella época, de suma importancia resultaron las jornadas de voluntariado en salud, que fortalecieron el trabajo en promoción y educación, con participación de trabajadores de la salud, trabajadores en general y pobladores de la ciudad y el campo [1].

Durante este periodo, las políticas públicas relacionadas con la modalidad de libre elección fueron reevaluadas, regulándose, en 1972, en el marco del Sistema Único de Salud. Esta situación incrementó la polaridad entre los partidarios de Allende y quienes observaban en la "vía chilena al socialismo" un grave riesgo para la integridad económica nacional y sus propios intereses, entre ellos, el Colegio Médico de Chile [1].

Quedaba así abierto el campo en disputa respecto de la salud pública, con los siguientes puntos: 1) aspiración de la salud como derecho consagrado vía políticas públicas; 2) reivindicación de la clase media respecto del cuidado y el derecho a elección del prestador de salud; 3) división de una salud para pobres y una salud de libre elección (Sermena), y 4) la idea de una institucionalidad privada como alternativa a la salud estatal [3].

El golpe cívico-militar encabezado por Augusto Pinochet en 1973 significó el freno al proceso de democratización de la salud. Como resultado, "se destruyeron los dos puntales de la política de salud del gobierno de la Unidad Popular: la democratización institucional de la salud y la creación de un sistema de salud sin clases" [1, p. 502]. La persecución, la tortura y el asesinato de personas comprometidas con la participación social tuvo su correlato en el campo de la salud.

En 1980 se sancionó la Constitución de la República de Chile (aún vigente), que reconoce como derecho la libertad de elección del usuario entre el sistema público y el privado. Con base en el principio de subsidiariedad del Estado, entiende que los servicios deben ser prestados en primera instancia por la sociedad civil, y en caso de que estos sean incapaces de prestarlos, el Estado debe asumir la responsabilidad [4]. Esto marcó una nueva concepción de los derechos sociales como objetos de mercado, y de la salud como bien transable. Entonces, ¿tiene cabida la noción de derecho social en el modelo de sociedad neoliberal en construcción?

Fue en el periodo dictatorial que comenzó a esbozarse la arquitectura del actual sistema de salud, con el Decreto con Fuerza de Ley núm. 3 [5]. En este, se crean tres subsistemas diferenciales: el público, a través del Fondo Nacional de Salud (Fonasa); el privado, con las Instituciones de Salud Previsional (Isapres), y un tercero, destinado a las Fuerzas Armadas.

Los años noventa, en Chile, estuvieron marcados por la recuperación de la democracia, que podría interpretarse ingenuamente como la recuperación de los valores y principios "suspendidos" y trastocados a partir del golpe de Estado de 1973. Sin embargo, podríamos preguntarnos: ¿se ha producido una ruptura de la noción mercantilista de la salud durante el periodo democrático?, y ¿se llega a producir una recuperación y resignificación de los derechos sociales?

La legislación y la arquitectura del sistema de salud siguen respondiendo a los lineamientos fijados por la dictadura. La trayectoria de las reformas de salud en Chile constituye una continuación de la reforma neoliberal implantada en la dictadura, siendo una de las más regresivas, mercantilizadas y privatizadoras de la región latinoamericana [6].

Luego de 44 años de continuidad de este proyecto político, y pensando que las posibilidades de transformación en salud parecieran supeditadas a la construcción (y la reconstrucción) de actores sociales portadores de un discurso contrahegemónico, consideramos necesario centrarnos en la perspectiva de los actores sociales que participan de acciones colectivas, a partir de explorar qué es lo que estos entienden por el derecho a la salud y la lucha por el mismo, en Chile.

Para responder esta pregunta, en esta investigación trabajamos con el concepto de representaciones sociales. Jodelet las define como "la manera en que aprehendemos los acontecimientos de la vida diaria, las características de nuestro medio ambiente, las informaciones que en él circulan, a las personas de nuestro entorno próximo o lejano" [7, p. 27]. Este conocimiento tiene como funciones: permite a los actores comprender el mundo, estructurar la valoración y la comunicación interpersonal, como también condiciona las prácticas [8].

Entonces, ¿por qué las representaciones sociales de las luchas por el derecho a la salud pueden ser diferentes en una misma sociedad? Este conocimiento social no es universal, está determinado por su contexto de construcción; de ahí que existan diferencias entre grupos sociales respecto a las representaciones sociales sobre los mismos objetos sociales. Es decir, que para poder abordarlas, debemos definir claramente el grupo social con el cual trabajamos y el contexto en el que este grupo se desarrolla. Así, en la presente investigación nos centramos en actores sociales que luchan activamente por el derecho a la salud en Santiago de Chile.

\section{Metodología}

Trabajamos desde la teoría fundamentada, es decir, una "teoría derivada de datos recopilados de manera sistemática y analizados por medio de un proceso de investigación. [...] la recolección de datos, el análisis y la teoría que surgirá de ellos guardan estrecha relación entre sí” [9, p. 21].

Abordar las representaciones sociales implica definir los presupuestos epistemológicos, las formas en que buscamos el conocimiento y los presupuestos 
ontológicos, e implica también establecer la naturaleza de nuestro sujeto/objeto de estudio [10]. Con base en esto, la aproximación al fenómeno se realizó desde el paradigma pospositivista, con enfoque cualitativo. Trabajamos con el método hermenéutico, indagando en las representaciones sociales a través de la interpretación de los discursos de actores sociales que forman parte de acciones colectivas por la salud. Parera Perez define discursos como "todas las formas de interacción hablada, formal e informal y todo tipo de textos escritos" que constituyen un medio para acceder al "universo simbólico y significante de los sujetos, constituido y constituyente de la realidad social" [11, p. 14].

La técnica utilizada correspondió a la entrevista focalizada, guía de preguntas abiertas construidas a partir de categorías preliminares, en relación directa con los propósitos planteados y la revisión bibliográfica. Esta guía sirvió para estructurar (no en forma cerrada) la entrevista, permitiendo profundizar en temas específicos y favoreciendo un conocimiento profundo. A partir de las entrevistas se incluyeron categorías emergentes. Las entrevistas fueron realizadas cara a cara, en ámbitos seleccionados por los entrevistados, y grabadas, previa autorización del informante, para su transcripción.

\section{Selección de informantes clave}

Para la selección de informantes se utilizó como criterio que hicieran parte activa de acciones colectivas por la salud en Santiago de Chile. En tanto se trabajó con la técnica de bola de nieve, se seleccionaron, en una primera instancia, dos informantes, con base en su trayectoria en la lucha por la salud y la relevancia de sus espacios de participación.

A partir de ellos se contactó al resto de los entrevistados, priorizando que formaran parte de diferentes espacios de lucha (gremios, organizaciones sociales, grupos políticos y red de usuarios).

El tamaño de la muestra fue determinado por el punto de saturación, entendiendo por este el momento en que "las nuevas entrevistas ya no aportan al proceso de comprensión de los patrones y relaciones sociales investigados" [12, p. 130]. En nuestra investigación, el punto de saturación se consiguió con nueve entrevistados, actores sociales que participan de acciones colectivas por la salud, ya que, como señala Parera Perez, "cuando los argumentos comienzan a repetirse se llega al límite de sujetos, pues una mayor cantidad de individuos no aporta ninguna significación diferente al contenido de la representación" [11, p. 16].

\section{Construcción metodológica}

Para desarrollar la investigación, trabajamos con el procedimiento planteado por Martínez [13]. A partir de la transcripción de las entrevistas y su codificación, se realizó la categorización ("derechos sociales", "ejercicio del derecho a la salud", "lucha social", "actores en lucha", "objetivos y repertorios de lucha", "resultados" y "referentes"). Integramos las categorías en estructuras particulares y generales, permitiendo dar sentido a las representaciones, entendiendo que "combinar la estructura con los procesos ayuda al analista a captar algo de la complejidad que forma parte tan importante de la vida" [9, p.139]. A partir de esto, se trabajó en la triangulación, relacionando los resultados con el marco teórico y antecedentes.

Cabe destacar que estas etapas no correspondieron a secuencias lineales, sino que estuvieron marcadas por avances y retrocesos, permitiendo la retroalimentación y la profundización en el proceso de construcción de conocimiento.

\section{Consideraciones éticas}

La presente investigación adhirió al código ético de la Asociación Internacional de Sociología [14], como también a las consideraciones éticas citadas por Pastor [15]. Por ello, para asegurar la participación libre e informada de los entrevistados, se les entregó un documento de consentimiento informado, previo a la entrevista. Allí se detallaron: los propósitos de la investigación; se describió la participación esperada; se solicitó autorización para el uso de grabadora; el tiempo requerido; el carácter voluntario y la posibilidad de abandonar la entrevista; los riesgos y beneficios; los criterios de anonimato y la información de contacto de los investigadores. Asimismo, se entregó el formulario de consentimiento informado para su firma y la copia correspondiente, con la firma de los investigadores.

\section{Resultados}

La información analizada se corresponde con entrevistas enfocadas a informantes clave seleccionados, nueve actores sociales que hacen parte activa de acciones colectivas por la salud en la ciudad de Santiago de Chile.

\section{Representaciones sociales acerca del derecho a la salud}

Para la interpretación del derecho a la salud construimos dos categorías: "Conceptualización de derechos sociales" y "Ejercicio del derecho a la salud en Chile".

* La convención utilizada significa "informante clave" (IC) y luego sigue la numeración. 
Los informantes clave definen los derechos sociales como condiciones básicas o mínimas que permiten el sostenimiento de la vida. Los más nombrados corresponden a vivienda, salud, educación y previsión. En sus palabras: "son las necesidades mínimas que deben ser satisfechas por parte del Estado y la sociedad en su conjunto [...], lo que hace posible vivir, y vivir dignamente" (IC3)."

En todos los casos se señala el rol del Estado como garante de los derechos sociales, y parte de los entrevistados precisó el rol de corresponsabilidad de la sociedad, en tanto capacidad de ejercer presión sobre el Estado y los gobiernos: "los derechos sociales son las obligaciones que tiene el Estado para con los ciudadanos [...]. Debería ser garante de ese acceso, con un papel de las comunidades y de los sindicatos y organizaciones sociales, en la elaboración y [el] diseño de los propios problemas que ellos identifiquen" (IC7).

Los derechos sociales en Chile son caracterizados por los informantes como fragmentados, desiguales y mercantilizados. Las políticas públicas se señalan como focalizadas en sectores priorizados, y consideran que esto producen altos niveles de desigualdad y fragmentación. En relación con la mercantilización, afirman que "el mercado privado tiene altos niveles de participación en el sistema educativo, de salud, previsional y de vivienda" (IC1).

A partir de estos rasgos, una de las informantes marcó que no es posible hablar de derechos sociales en Chile: "aquí en Chile no tenemos derechos sociales como tales, en Chile el Estado no es garantista [...] derechos sociales no existen" (IC4), mientras que el resto de los participantes dijeron que existen, pero restringidos.

Al momento de definir la salud, la mayoría de los entrevistados utilizó términos relacionados con el concepto de la Organización Mundial de la Salud, plasmado en la Constitución de 1948, que la define como "un estado de completo bienestar físico, mental y social, y no solamente la ausencia de afecciones o enfermedades" [16]. Enfatizaron la necesidad de superar la visión de la salud como proceso salud-enfermedad individual y entenderla como parte de un todo social, al decirnos: "salud es hablar de la vida, mantener y desarrollar la vida en las mejores condiciones posibles, eso es salud. Por lo tanto, salud tiene que ver con todo, tiene que ver con las relaciones humanas, trabajo, vivienda, y en último término con las enfermedades, pero pasa por todo [...]" (IC3).

Al hablarnos sobre el ejercicio del derecho a la salud, los informantes hicieron hincapié en tres aspectos: marco constitucional, construcción histórico-social y sistema de salud. Acerca del primero, expresaron: "nuestra Constitución Política no garantiza el derecho a la salud, lo deja establecido claramente; lo que te garantiza es el derecho al acceso a la salud [...] no lo establece como derecho fundamental" (IC1).

Con respecto a la construcción histórico-social, los informantes señalaron tres etapas históricas: el gobierno de la Unidad Popular (1970-1973), la dictadura militar (1973-1990) y el periodo democrático (1991-2018).

Al referirse al gobierno de la Unidad Popular, se resaltó la figura de Salvador Allende y su importancia en la construcción de un proyecto político con especial énfasis en la salud. Dicho Gobierno fue definido por los participantes como una etapa donde se redujeron drásticamente los índices de mortalidad por causas infecciosas y la desnutrición infantil. Se destacaron los avances en infraestructura urbana y la implementación de planes y programas gubernamentales, con amplia participación comunitaria y de grupos políticos de izquierda con fuerte arraigo territorial, que tuvieron como resultado, según los informantes, rápidos avances en las condiciones de salud de la población a partir del trabajo articulado entre el Estado y las comunidades.

La gente se organizaba y su motivación era la salud y logramos construir 80 comités de salud en ese pueblito, de tal manera que la propia población estaba atenta de cuando nacía un niño, una mujer quedaba embarazada; y cuando había un problema de salud, ellos sapeaban ${ }^{\dagger}$ o llevaban a la gente al hospital, tuvimos resultados fabulosos. Toda la gente en 80 comités, 700 personas organizados en el tema de la salud, fue impresionante. Éramos una organización social viva [...]. Después vino la represión terrible y se perdió todo (IC3).

El golpe de Estado de 1973 fue calificado como un quiebre histórico en el desarrollo del derecho a la salud en Chile. En este sentido, señalan el carácter "brutal" de la violencia ejercida por el Gobierno dictatorial, dirigida a acallar las formas de participación social, y las resistencias a la implementación del modelo neoliberal en el diseño y la construcción de la arquitectura del sistema de salud chileno, destacando que, producto de este modelo, se dio la la mercantilización del acceso a la salud, y la consecuente y progresiva creación de grupos económicos en torno a la prestación de servicios.

[...] con la dictadura se destruyó todo y ahora todo es privado [...]. Para callarnos fue necesario un golpe de Estado, que fue brutal, que torturó, mató y desapareció a todos los que participaban [...] la verdad es que la dictadura hizo muy bien la pega [el trabajo] [...], nos implantó un sistema neoliberal a la fuerza, que hace creer a la gente que los problemas se resuelven de a uno, sin la comunidad, pero no es así [...] (IC5).

\footnotetext{
"Sapear": delatar; aquí se usa en tono jocoso.
} 
El nuevo periodo democrático, iniciado en 1990, no representó para los entrevistados un cambio de modelo económico-político, sino, más bien, la profundización del modelo neoliberal. Para ellos implicó, en el campo de la salud, mayores niveles de privatización y mercantilización. Los participantes señalaron que el Estado solo asegura el acceso a la salud con base a un "modelo subsidiario y de focalización de gastos" (IC2), implementado durante la dictadura.

Acerca del sistema de salud, los informantes hicieron énfasis en que la libre elección se encuentra determinada por la capacidad adquisitiva, marcando un acceso desigual. El sistema de salud es percibido como fragmentado, mercantilizado y desigual, en tanto existen dos subsistemas diferenciados en recursos y calidad de la atención: Isapres y Fonasa.

El subsistema público (Fonasa) es percibido como "desfinanciado, con atención limitada por los escasos recursos materiales, infraestructura deteriorada, falta de equipamiento y camas hospitalarias, falta de médicos especialistas y escases de medicamentos [...]". Se resalta que, como consecuencia de esta falta de recursos, se generan "listas de espera para la atención", consideradas "el resultado de la crisis del sistema de salud en Chile, que lleva muchos años, pero ya es insostenible" (IC2). Además, las medidas para afrontar las listas de espera por parte del Estado son calificadas como "insuficientes, en tanto resultan medidas administrativas que no resuelven la problemática de base [...], llaman a la persona por teléfono y si no atendió, la borran de la lista, y la lista de espera ya pasó [...]" (IC1). También se destaca el acceso desigual, al diferenciar entre patologías y grupos etarios o de sexo del usuario. "El Plan AUGE" te dice que tú te puedes enfermar de las 85 enfermedades que te cubre el plan [...]. Cuando una persona se muere porque no hay atención médica oportuna [...] un examen que debe hacerse y el hospital no puede asegurarlo y la persona no tiene dinero y se muere [...], una experiencia que yo conozco de cerca [...], ahí la desigualdad se ve de frente, en toda su magnitud" (IC6).

Sobre las formas de acceso a la salud, los entrevistados señalaron que la comunidad ha desarrollado estrategias para superar las barreras de acceso, resaltando que estas son reactivas y puntuales, y no se constituyen en formas de organización posteriores para resolver la problemática. Un ejemplo es "la organización de 'bingos' o 'rifas' en las comunidades, que permiten recaudar fondos para el pago de medicamentos o prestaciones de salud requeridos por un vecino y que no pueden ser afrontados económicamente" (IC1). Otra de las estrategias desarrolladas es acudir a la urgencia hospitalaria para sortear la lista de espera; "a veces la gente prefiere, cuando lleva mucho tiempo en lista de espera, por ejemplo, por cirugía de cadera, espera estar mal mal y se va a la guardia de urgencia y ahí tiene una posibilidad de ser operado en cirugía de urgencia [...] pero no siempre pasa; a veces, el médico dice que no, y es no [...], y siempre que haya médico especialista que lo pueda operar. Si es no, te devuelven a tu casa" (IC4).

Con respecto a los responsables de asegurar el derecho a la salud y las posibilidades de acceso, señalaron dos actores fundamentales: el Estado y la comunidad organizada. Al Estado se le otorgó un rol protagónico como garante de derechos, articulador de demandas, y responsable de asegurar la calidad y la accesibilidad al sistema de salud de la población en su conjunto. A la comunidad, se le hace corresponsable, en tanto actor organizado que es capaz de enfrentar, a partir de la presión, las falencias del sistema.

Al ser consultados sobre un tercer actor, los sectores privados de salud, señalaron que este no tendría responsabilidades en torno al derecho a la salud, en tanto el objetivo que persiguen corresponde al lucro. $\mathrm{Su}$ participación en el sistema de salud es vivida como negativa, en tanto se considera que "la enfermedad es un negocio [...], se enriquecen a costa de nosotros, las sociedades médicas que tienen una especie de tríada con grupos económicos, como todo lo que se lucra en Chile" (IC3).

La amplia participación de los sectores privados en salud es atribuida al Estado, responsable de la forma como se desarrolla la actividad comercial en salud, y la fortaleza que estos sectores tienen. "La mercantilización de la salud en un estado patronal, que todo lo que se hace es para ganancia de algunas empresas [...] el pueblo pobre no tiene posibilidad de acceso a la salud especializada y de calidad a la que solo accede el $20 \%$; el $80 \%$ no accede" (IC1).

\section{Representaciones sociales acerca de las luchas por el derecho a la salud}

Para la interpretación de la lucha por el derecho a la salud construimos cinco categorías: "Lucha", "Actores sociales", "Repertorios de lucha", "Resultados" y "Referentes".

La lucha por la salud fue relacionada con la lucha por la vida. "Es la vida, yo pienso... es luchar por la vida. La urgencia de implementar lucha, de hacer acciones, llevar al debate de qué salud queremos, es súper urgente" (IC2).

\# El Plan de Acceso Universal de Garantías Explícitas en Salud (auge-ges) se regula por la Ley 19966 (2004). Tiene por objeto asegurar la atención médica de usuarios bajo criterios de acceso, oportunidad en la atención, calidad y protección financiera en un número determinado de enfermedades (80 patologías para 2019). Esta cobertura incluye a usuarios de Fonasa e Isapres 
En las luchas desarrolladas, los informantes identificaron cuatro actores sociales centrales en Santiago: gremios, usuarios, estudiantes y grupos políticos de izquierda. Los entrevistados los definieron diferencialmente en cuanto a su participación: los gremios fueron considerados actores centrales, dada su participación histórica en torno a las reivindicaciones laborales, la ampliación del financiamiento y las denuncias de falencias en el sistema de salud. Se los describió como altamente fragmentados, entendiendo que la inexistencia de un sindicato único o de niveles de articulación entre los distintos gremios favorece la fragmentación en el campo de los trabajadores, de acuerdo con profesiones, lugares de trabajo y la relación de los gremios con partidos políticos y la institucionalidad: "los gremios de la salud son fuertes, pero están muy fraccionados, aunque hay sectores que están luchando fuerte [...] son los más importantes, aunque principalmente llevan temas de reivindicaciones laborales, pero también son los que hacen presión al Estado para que mejore la infraestructura" (IC2).

Con respecto a los usuarios, se reconoció el rol histórico y la importancia de las organizaciones comunitarias durante los periodos previos al golpe de 1973, mientras que en la actualidad es considerado el actor más débil, por sus bajos niveles de articulación: "usuarios que nos hemos ido encontrando en algunas luchas, pero nos falta [...], nos falta organización, organización a los usuarios y organización a los trabajadores, pero la veo incipiente" (IC8).

Acerca de los estudiantes, se describió su participación, en forma articulada, con las luchas por la educación pública y gratuita, al expresar: "La lucha por una educación gratuita y de calidad y la salud gratuita y de calidad van muy ligadas, y eso se está dando, hay hartos estudiantes en la lucha por la salud" (IC7). En relación con cómo perciben estas luchas, nos dicen: "Estamos también los estudiantes, pero muy fragmentados ¿no?, en una etapa inicial, pero ahí confluyen sectores de pacientes, población, trabajadores de la salud y jóvenes de izquierda" (IC7).

Con respecto a los grupos políticos de izquierda, señalaron que si bien la lucha por el derecho a la salud no constituye el temario central, sí es un ámbito de importancia en un proyecto de transformación políticoeconómico general. Como afirma un estrevistado, "no es luchar por más remedios y más hospitales; es una lucha por una sociedad diferente" (IC6).

A los actores sociales se le asignan tres roles fundamentales: educativo, difusión de problemáticas en salud, y articulación, con énfasis en el ámbito territorial.

Sobre las instancias de coordinación entre actores sociales se mencionó a la organización Movimiento Salud para Todos (MSPT), caracterizándolo como un nuevo espacio: "Recién en 2015 comenzó una organización que está tratando de articular la lucha de trabajadores, estudiantes y comunidad, pero eso está recién partiendo, como la idea de todos trabajar juntos; ese es el Movimiento Salud para Todos [...], que yo creo que hay que apuntalar y apoyar, participar de una u otra manera, pero va a ser un proceso largo" (IC2). Al consultar a los participantes del MSPT, estos se autodefinieron como "espacio de coordinación", reconociendo que su trabajo se encuentra en una fase inicial.

Acerca de los objetivos de la lucha, se distinguieron tres tipos:

1. Objetivos de corto plazo, necesarios para lograr mejoras en salud en el marco del sistema actual (reivindicaciones laborales, financiamiento e inversión en infraestructura).

2. Objetivos de mediano plazo, en los que se prioriza la construcción de un "nuevo modelo" de salud, pendiente en su formulación, que fue identificado como central para el fortalecimiento de la lucha y la coordinación entre actores sociales.

Con respecto a este nuevo modelo, los entrevistados mencionaron ciertos rasgos comunes: sistema único de salud de cobertura universal, con énfasis en la atención primaria y gestionado por los trabajadores y los usuarios; gratuito y de calidad, reconociendo que "todavía no se ha logrado mucho, porque todo lo que parecía solución termina siendo perfeccionamiento del sistema y más privatización". Así mismo, señalaron que un factor fundamental de la construcción de este nuevo modelo es la participación activa, ya que se no se pretende "un modelo creado por expertos" (IC7).

3. Objetivo de largo plazo, centrado en un cambio de modelo económico y político general, que posibilite un nuevo modelo en salud. Por ello, se consideró que la lucha en última instancia es por una nueva sociedad, siempre desde la certeza de que esto "llevará años y años", y que la construcción debe contemplar que hay una tarea urgente, la de ir "solucionando necesidades desde hoy" (IC7).

Cabe mencionar que las tareas para el desarrollo de estos objetivos no fueron planteadas como una secuencia lineal, sino como un proceso dinámico que se construye en permanente relación de actores sociales, Estado y organizaciones sociales, en un contexto histórico-social cambiante.

Los repertorios de las acciones colectivas fueron definidas y diferenciadas de acuerdo con urgencias y necesidades, planificadas puntualmente en torno a la problemática y el objetivo; "temas concretos como el financiamiento de la atención primaria ha sido un tema de movilización constante nuestra [...], nos movilizamos 
durante muchos años atrás ehhh, y ha tenido un aumento considerable" (IC1).

En general, las acciones que mencionaron estuvieron dirigidas a dos actores sociales: el Estado y la sociedad. Se buscó, por un lado, ejercer presión sobre el Estado y, por otro, difundir a la sociedad una situación determinada. "Han sido buenas [las acciones], la gente está empezando a despertar, pero somos pocos, tenemos que ser hartos para ejercer una real presión al Estado y al poder financiero que nos gobierna" (IC3).

La distinción entre Estado y Gobierno, así como la identificación de actores económicos como parte de la lucha emergen tímidamente, todavía subsumida esta última en la contingencia del corto plazo, y en la conveniente confusión-superposición entre Estado y Gobierno, instalada en el imaginario colectivo. Matamala lo expone claramente, al relatarnos que "para el abogado y experto en lobby Renato Garín, el Chile actual es un caso de corporativismo, o sea un acuerdo entre un grupo de privilegiados en que el Estado trabaja para ellos", citando, además, a los abogados Bosselin y Briones, quienes describen a Chile como "una democracia controlada en las sombras por el dinero", por quienes detentan el poder económico; "tienen el músculo, tienen razones para usarlo [...] y no tienen restricciones para no hacerlo [...], para prevenir que el dinero influya en las políticas públicas" [17, p. 26].

Los informantes señalaron que no solo es importante la visibilización de las acciones, sino también los contenidos; por ello resulta clave la comunicación, destacando que existe una relación compleja, ya que los medios fomentan la noción de una relación de oposición dicotómica entre trabajadores y usuarios. Los actores sintieron mayor apoyo a través de medios alternativos de comunicación o estrategias de tipo callejera: "No se pone ese tema sobre el tapete, los medios no abren espacio para dejar en claro esa cuestión que para nosotros es fundamental [...], los que luchamos quedamos como invisibles, ves la tele y nada [...], dependemos de los medios alternativos" (IC9).

En cuanto a los repertorios, podemos señalar que se desarrollan en forma simultánea repertorios no contenciosos (reuniones o negociaciones desarrolladas por gremios), y contenciosos (paros, funas - denuncias públicas-, marchas y tomas desarrollados por todas las organizaciones participantes). Se resalta que el MSPT es la organización que ha desarrollado los repertorios más novedosos en el ámbito de las luchas por la salud y con mayor visibilidad: "se ha hecho ocupación de espacios, tanto ministerios como Superintendencia de Salud, panfletos, mitines, denuncias en urgencias, marchas [...], lo demás son congresos y encuentros [...], se ha funado a la ministra, a la [Michelle] Bachelet, al director del servicio [...] algunas espontáneas, otras planificadas
[...], o sea, donde se pueda, hemos hecho algo, lo humanamente posible [...], sí, las acciones pueden pensarse que son medias locas [un poco locas], pero qué vamos a hacer" (IC3).

Otro aspecto de la visibilización es la capacidad de estos grupos para instalar temas en la agenda política, destacando como un logro que los candidatos a las elecciones presidenciales de 2017 abordaran la crisis del sistema de salud a partir de dos de sus consecuencias más visibles: listas de espera y concesiones hospitalarias.

Se señalaron tres tipos de resultados o logros considerados "concretos", y que podrían enmarcarse en los objetivos de corto plazo: 1) incremento en el financiamiento y la ampliación de las enfermedades incluidas en el Plan AUGE-GES, que permitieron la ampliación de servicios y prestaciones por parte del subsistema público; 2) la progresiva instalación de nuevas ideas tendientes a romper con la naturalización del sistema de salud vigente, y 3) la capacidad de articular diferentes actores sociales en un trabajo interorganizacional.

En cuanto a los referentes en torno a la lucha social, se destacaron: el movimiento estudiantil y el movimiento $\mathrm{NO}^{+} \mathrm{AFP}$ (Administradoras de Fondos de Pensiones), por su capacidad de articular numerosas organizaciones; la amplia difusión de las acciones; la territorialización; la presión ejercida sobre el Gobierno y la inclusión de sus objetivos en la agenda pública. Asimismo, se mencionó las luchas desarrolladas por comunidades indígenas (principalmente la mapuche) en torno al rescate y la valorización de la cultura ancestral como una forma de romper con la visión medicalizada y mercantilizada de la salud.

\section{Discusión}

El derecho a la salud es considerado, por los entrevistados, una necesidad básica para el sostenimiento de la vida. Para el caso chileno, es definido por su acceso desigual, fragmentación y mercantilización. Estas características se deben a que la salud, como parte del todo social, se encuentra subsumida en un modelo político-económico capitalista de tipo neoliberal. Este modelo entiende la salud como responsabilidad individual, que, según Ferrer, "legitima la aplicación de las políticas neoliberales en el sistema de atención de la salud y la privatización instalada por la dictadura" [18, p. 10]. En contraposición, los actores sociales que luchan y que fueron consultados sostienen que el Estado es responsable de asegurar el acceso, y la sociedad organizada, de ejercer presión para su cumplimiento.

Cobra especial importancia la construcción histórico-social del sistema de salud, a partir del cual se explica la situación actual, donde se destacan dos 
periodos diferenciales: previo al golpe cívico-militar, donde la figura de Allende es central, y posterior a 1973, con la implementación del modelo neoliberal.

Resaltamos el sentido de continuidad que se le otorga en la democracia al periodo iniciado en 1973, al considerar, en concordancia con Tetelboin [19], que el retorno a la democracia significó una profundización del modelo neoliberal, significando mayores niveles de privatización y mercantilización de los derechos sociales.

La lucha por el derecho a la salud es considerada central y urgente, y alude a la defensa de la vida en forma integral. Los objetivos de lucha son diferenciados en corto plazo (reivindicaciones laborales, financiamiento e inversión) y que acaparan los esfuerzos actuales; mediano plazo (nuevo modelo de salud) y largo plazo (nuevo modelo económico-político).

En cuanto a los participantes, los entrevistados señalan cuatro actores sociales centrales: gremios, estudiantes, usuarios y grupos políticos de izquierda. Estos son caracterizados por bajos niveles de articulación y adhesión, lo que se explica como consecuencia de la violencia ejercida durante la dictadura. Asimismo, se resalta que son las organizaciones de usuarios las más débiles en cuanto a acción y participación, refiriendo que sus acciones aluden principalmente a prácticas tendientes a solucionar problemas individuales.

Los repertorios desarrollados corresponden a dos tipos, de acuerdo con Cohen [20]: repertorios no contenciosos (negociaciones, reuniones) $\mathrm{y}$, en forma simultánea, contenciosos (paros, funas, marchas y tomas). Estos repertorios se articulan con objetivos de corto plazo, que tienen como interlocutor principal al Estado [21]. Sin embargo, resaltamos la importancia que toma la sociedad como interlocutora, manteniendo el foco puesto en el Estado-Gobierno, y no en quienes parecen controlarlos.

A partir de los repertorios contenciosos desarrollados, es posible observar la influencia que tiene el movimiento de estudiantes, ya que se toman repertorios propios de esta lucha (tomas de instituciones y funas), mostrando la confluencia existente entre los diferentes campos de lucha.

Por lo dicho, consideramos que las representaciones acerca de las luchas por el derecho a la salud de los actores sociales nos permiten situarlas, de acuerdo con Moscovici, como representaciones emancipadas. Estas representaciones "no tienen el alcance macro de las hegemónicas. Son propias de grupos que surgen con nuevas visiones y criterios del mundo, trayendo consigo la semilla de un posible cambio. En esencia, éstas son muy flexibles y dinámicas" [22, p. 154].

Al contrastar nuestros resultados con los de Mossos y Mora [21], sobre los elementos nodales del discurso en el Movimiento Social por la Salud, y sus retos y posibilidades políticas en el contexto posterior a la Constitución de 1991 en Colombia, observamos aspectos compartidos (denuncias sobre la mercantilización de los derechos sociales y amplitud para incluir, en la lucha, diferentes grupos sociales) y diferencias. Estas últimas aluden a la incapacidad, en el caso chileno, de articular un movimiento de carácter nacional.

Resaltamos que las representaciones de los actores, en el caso chileno, resultan concordantes, a pesar de hacer parte de diferentes espacios de participación, y esto puede constituir una base importante para la conformación y la consolidación de un movimiento a nivel nacional. El MSPT podría constituirse en un espacio estratégico; por ello, su estudio puede ser de interés, en cuanto a las posibilidades que contiene en la conformación de un espacio de lucha integrador y de alcance nacional.

\section{Conclusión}

Los actores que hacen parte de las acciones colectivas no conforman un movimiento unificado ni estructurado. Entre las dificultades para la participación y la constitución de un movimiento de carácter nacional podemos observar los temores que persisten en la sociedad, secuelas de la represión sistémica y sistemática ejercida por la dictadura, y que parecen determinar esta conducta.

Las luchas por el derecho a la salud constituyen un fenómeno emergente, que puede enmarcarse en un proceso de repolitización de la sociedad chilena, en cuyo seno las representaciones acerca de las luchas por el derecho a la salud de los actores sociales investigados tendrían un carácter emancipatorio, con base en su perfil contrahegemónico.

Consideramos que el proceso dialéctico de conquista del derecho a la salud sigue poniendo su foco en el Estado como interlocutor, invisibilizando de manera cómplice y conveniente a los actores privados del complejo médico industrial, que a su vez hacen uso del Estado como dispositivo distractor, especie de mampara para contener las exigencias de la población, no solo por la salud, sino también por el conjunto de derechos sociales que hacen digna y posible la vida. Estos actores logran apropiarse del Estado por la fuerza, y ya consolidados instalan una "democracia" a la medida de sus intereses. La tarea es hacer visible y enfrentar a los constructores y beneficiarios de este perverso sistema, fundado en la desigualdad y la injusticia. La tarea parece ser la descolonización del Estado y de la vida.

A modo de propuesta, resaltamos la necesidad observada de (re)pensar herramientas teórico-metodológicas para la conceptualización de la salud. La utilización de conceptos cercanos al modelo de la organización en torno a la salud existente se contrapone y limita la perspectiva contrahegemónica que portan los actores; por ello, sería de utilidad discutir las bases conceptuales metodológicas y las experiencias sobre la salud colectiva, como proyecto político superador.

En últimas, es importante contrastar las luchas por la salud como derecho, con la experiencia y con los logros de los pueblos originarios, mediante su participación 
orgánica en la discusión del artículo 7 de la Ley de Salud [23] relativo a las prácticas interculturales.

\section{Financiación}

Esta investigación fue financiada con recursos propios.

\section{Declaración de conflicto de intereses}

Los autores no tienen ningún conflicto de intereses.

\section{Declaración de responsabilidad}

Se declara que los puntos de vista expresados son responsabilidad de los autores.

\section{Referencias}

1. Illanes Oliva M. En el nombre del pueblo, del Estado y de la ciencia Historia social de la salud pública. Chile: Ministerio de Salud; 2010.

2. Tapia Olmos J. Revisión histórica y legislativa del derecho a la salud en Chile [Memorias para optar al grado de licenciado en Ciencias Jurídicas y Sociales] Santiago de Chile: Universidad de Chile [internet]; 2015 [citado 2017 jun. 5]. Disponible en: http://repositorio. uchile.cl/bitstream/handle/2250/130057/Revisión-histórica-ylegislativa-del-derecho-a-la-salud-en-Chile.pdf;sequence=1.

3. Merino C. Del proyecto interrumpido de la democratización de la salud a la práctica sanitaria neoliberal en Chile. Chile: Colección monográficos Universidad de Los Lagos; 2016.

4. Allard Soto R, Henning Leal M, Galdamez Zelada L. El derecho a la salud y su (des)protección en el Estado subsidiario. Estudios Constitucionales. 2016; 14(1):95-138.

5. Chile, Ministerio de Salud. Decreto con fuerza de Ley núm. 3, por el cual se fija normas para el otorgamiento de prestaciones y beneficios de salud, por instituciones de salud previsional [internet]. (1981 abr. 27) [citado 2017 may. 26]. Disponible en: http://www.leychile.cl/Navegar?idNorma=3570.

6. Parada Lezcano M, Ibacache Carrasco C, Santana Nazarit P, et al. Informe de salud en Chile. En: Espinoza E. La lucha por el derecho a la salud en América Latina. San Salvador: Asociación Latinoamericana de Medicina Social Movimiento por la salud de los pueblos; 2014. pp. 31-51.

7. Jodelet D. La representación social: fenómenos, conceptos y teoría. En: Moscovici S. Psicología social II. Pensamiento y vida social. Psicología social y problemas sociales. Barcelona: Paidós; 1986. pp. 478-94.

8. Sandoval C. Programa de especialización en teoría, métodos y técnicas de investigación social. Colombia: Instituto Colombiano para el Fomento de la Educación Superior; 2002.

9. Strauss A, Corbin J. Bases de la investigación cualitativa. Técnicas y procedimientos para desarrollar la teoría fundamentada. Medellín: Editorial Universidad de Antioquia [internet]; 2002 [citado 20171 jul. 8]. Disponible en: https://diversidadlocal.files. wordpress.com/2012/09/bases-investigacion-cualitativa.pdf.

10. Araya Umaña S. Las representaciones sociales: ejes teóricos para su discusión. Costa Rica: Facultad Latinoamericana de Ciencias Sociales; 2002.
11. Perera Perez M. A propósito de las representaciones sociales: apuntes teóricos, trayectoria y actualidad. Centro de Investigaciones Psicológicas y Sociológicas. [internet]; 2003 [citado 2018 jun. 13]. Disponible en: http://biblioteca.clacso.edu. ar/Cuba/cips/20130628110808/Perera_perez_repr_sociales.pdf.

12. Breilh J. Nuevos conceptos y técnicas de investigación. En: Villasana P. Los núcleos de atención primaria. Una evaluación crítica a doce años de su implementación en el Estado Aragua. [Trabajo presentado ante el Departamento de Salud Pública para ascender a la categoría de profesor asociado]. República Bolivariana de Venezuela: Facultad de Ciencias de la Salud, Escuela de Medicina "Witremundo Torrealba"; 2003.

13. Martínez Miguelez M. La investigación cualitativa (síntesis conceptual). Revista IIPSI [internet]; 2006 [citado 2017 jul. 10]; 9(1):123-46. Disponible en: http://sisbib.unmsm.edu.pe/ bvrevistas/investigacion_psicologia/v09_n1/pdf/a09v9n1.pdf.

14. Asociación Internacional de Sociología. Código ético de la Asociación Internacional de Sociología. Madrid: Universidad Complutense [internet]; 2001. [citado 2017 jul. 20] Disponible en: https://www.isa-sociology.org/es/sobre-isa/codigo-etico-440.

15. Pastor Montero S. La ética en la investigación cualitativa en salud. Arch Memoria [internet]. 2011; 8(4) [citado 2017 jul. 10]. Disponible en: http://www.index-f.com/memoria/8/8400.php.

16. Organización Mundial de la Salud. Constitución de la Organización Mundial de la Salud. New York; [internet]; 1948. [citado 2017 jul. 26]. Disponible en: https://www.who.int/governance/eb/who_ constitution_sp.pdf.

17. Matamala D. Poderoso caballero. El peso del dinero en la política chilena. Santiago de Chile: Ediciones Catalonia Periodismo UDP; 2015.

18. Ferrer M. ¿La salud como responsabilidad individual? Análisis del concepto de salud en los Programas de Gobierno de la Concertación en Chile. Acta Científica del XXIX Congreso de la Asociación Latinoamericana de Sociología 2013 ISBN 978-956-19-0828-4.

19. Tetelboin C. Tendencias y contratendencias en el sistema de salud de Chile en el marco de la situación regional. En: Tetelboin C, Laurell AC. Por el derecho universal a la salud. Una agenda latinoamericana de análisis y lucha. Chile: Clacso, 2015. pp. 75-98.

20. Cohen J, Touraine A, Melucci A, et al. Teoría de los movimientos sociales. En: Echeverry-López M, Borrero-Ramírez Y. Protestas sociales por la salud en Colombia: la lucha por el derecho fundamental a la salud, 1994-2010; 2015. pp. 354-64.

21. Mossos Jiménez MA, Mora Lemus G. Movimientos sociales subalternos: análisis crítico del discurso del Movimiento Nacional por la Salud y la Seguridad Social en Bogotá. Revista Controversia [internet]. 2015 [citado 2017 jun. 13]; (204):47-75. Disponible en: http://www. revistacontroversia.com/index.php?journal=controversia\&page $=$ article \&op=view\&path $\% 5 \mathrm{~B} \% 5 \mathrm{D}=184 \&$ path $\% 5 \mathrm{~B} \% 5 \mathrm{D}=$ pdf_ 10 .

22. Rubira-García R, Puebla Martínez B. Representaciones sociales y comunicación: apuntes teóricos para un diálogo interdisciplinar inconcluso. Revista Controversia [internet]. 2018 [citado 2019 jun. 13]; 147-67. Disponible en: http://www.scielo.org.mx/pdf/ conver/v25n76/2448-5799-conver-25-76-00147.pdf, DOI: https:// doi.org/10.29101/crcs.v25i76.4590.

23. Chile, Ministerio de Salud Ley de Salud. Ley 20584. Regula los derechos y deberes que tienen las personas en relación con acciones vinculadas a su atención en salud [internet] (2002 abr. 24). [citado 2017 may. 26]. Disponible en: https://www.leychile. cl/Navegar?idNorma $=1039348$. 